\title{
The role of arthrography in the management of dysplasia epiphysealis hemimelica
}

\author{
Angelena M.W. Ho, M.B.B.S., Caroline E. Blane, M.D., and Thomas F. Kling, Jr., M.D. \\ Departments of Radiology (Section of Pediatric Radiology) and Surgery (Section of Pediatric Orthopedics) \\ at the University of Michigan Medical Center, Ann Arbor, Michigan, USA
}

\begin{abstract}
Dysplasia epiphysealis hemimelica can be diagnosed on plain radiographs of the affected areas. However, double contrast arthrography in three new cases provided additional information. The cartilaginous portions of the lesion at the articular surface of the bone were precisely demonstrated, aiding the orthopedic surgeon in deciding which patients should have surgery and planning the extent of operation necessary.
\end{abstract}

Key words: Arthrography - Dysplasia epiphysealis hemimelica - Trevor disease

Dysplasia epiphysealis hemimelica (DEH) is a rare disorder affecting the epiphysis, causing overgrowth on one side, usually the lower limb, particularly at the knee and ankle $[9,12]$. Presentation may be a noticeable swelling, pain, or a valgus or varus deformity. Plain radiographs usually reveal the underlying abnormality, depending on the amount of calcification. The management of these cases can be difficult. We present three new cases of DEH in which arthrography played a role in planning surgical management.

\section{Materials and methods}

The three boys were aged 2 years, 3 years, and 14 years at presentation. One patient was seen at another institution. All the children had plain radiographs of the involved extremity, hand films for bone age assessment, and double contrast arthrography. The arthrograms were carried out by the standard method [6]. Following sterile preparation, the knee was injected with $4 \mathrm{cc}$ of Renografin $76 \%$ and $10-50 \mathrm{cc}$ of air. The ankle had $2 \mathrm{cc}$ of contrast and $5 \mathrm{cc}$ of air injected. Multiple spot films

Address reprint requests to: Angelena Ho, M.D., Department of Radiology, University of Michigan, Ann Arbor, MI 48109 , USA and standard overhead radiographs of the joint were made. The youngest child also had computed tomography of the knees.

\section{Results}

Two children presented with masses at the knee causing a valgus deformity. In the youngest child the abnormality was noted in infancy and the affected leg was longer than the normal. The plain radiographs show the typical calcification seen in DEH (Figs. 1 and 2). The ossification centers of the medial cuneiform and navicular of the left foot on the involved side in one patient were also abnormal (Fig. 1D). Double contrast arthrography demonstrated the intra-articular extent of the lesion, the configuration of the cartilaginous articular surface, and the mechanics during movement under fluoroscopy (Figs. 1 E and 2B). Computed tomography of the knees in one case confirmed the calcified mass to be within the unossified cartilage of the left distal femoral condyle (Fig. 1F).

In the third case, the child presented with a mass at the ankle (Fig. 3). The affected limb was shorter than the normal. The other bones involved were the left navicular, left medial cuneiform, middle phalanx of both right and left third toes, and proximal left tibial metaphysis (Fig. 3C). The double contrast arthrogram showed the smooth articular surface and cartilaginous extent of the lesion (Fig. 3D). Surgical resection in all of these cases has corrected the growth abnormalities at this time. Long-term follow-up will be necessary to determine if regrowth of the abnormal cartilage continues.

\section{Discussion}

$\mathrm{DEH}$ is a developmental disorder of epiphyseal growth causing osteocartilaginous proliferation at 

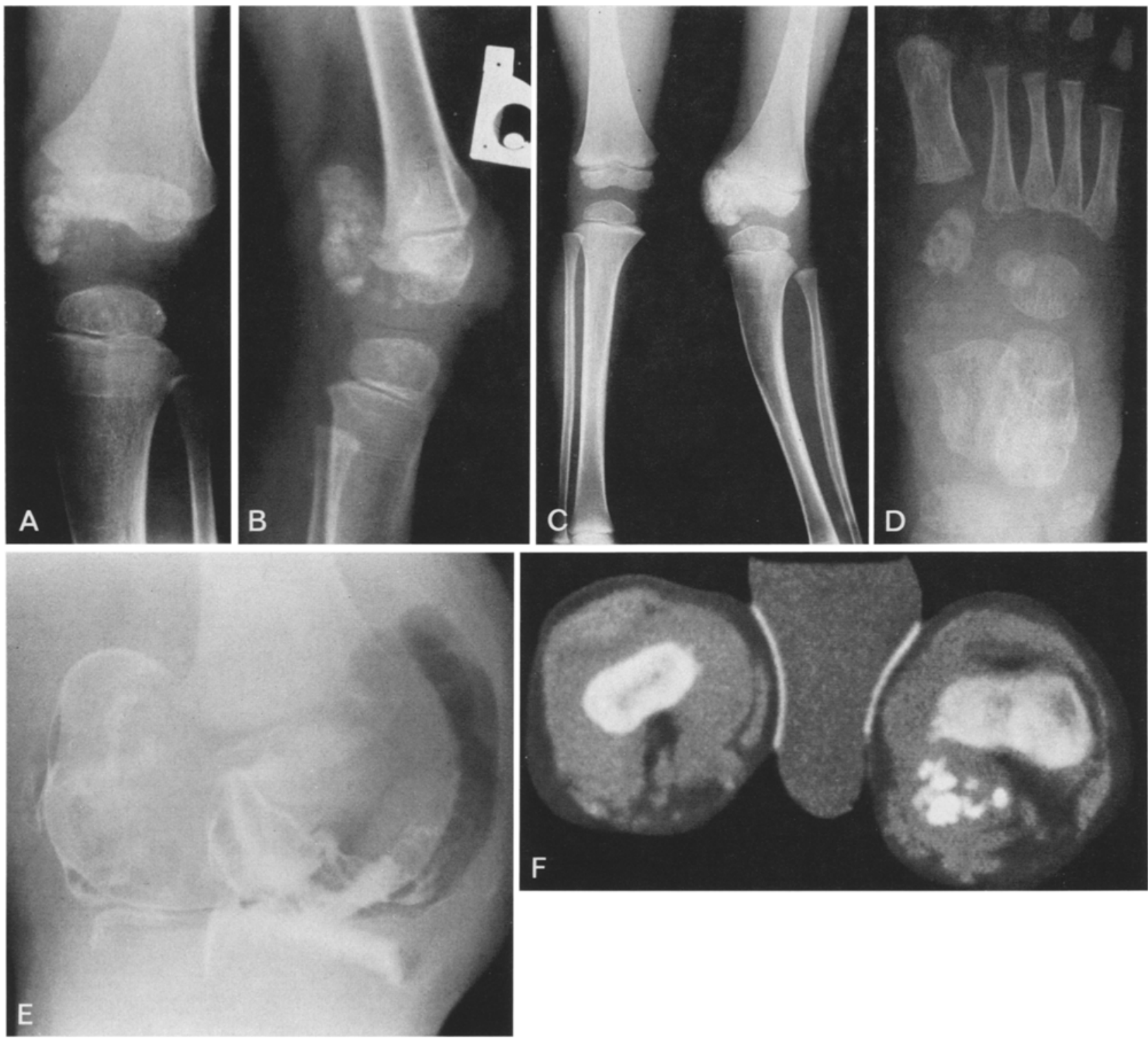

Fig. 1. A, B Anteroposterior (AP) and lateral views of the left knee in a 2-year-old boy with valgus deformity. A large irregular calcified mass arises from the medial femoral condyle. C Note lengthening of affected leg and medial bowing of left tibia at age four years. D Radiograph of the left foot shows involvement of the medial cuneiform and navicular. E Oblique view of the knee during arthrography outlines a smooth articular surface of affected femoral condyle and uninvolved tibial condyles. F CT scan of the distal femurs showing calcified mass within unossified cartilage of posterior aspect of medial condyle

one side of the epiphysis. The commonest sites involved are the talus, the distal femoral medial condyle, the distal tibial medial condyle, and the proximal tibial epiphysis, navicular, and medial cuneiform, in that order $[9,12]$. The age of the patient is usually between 2 and 14 years [9]. Males are affected far more frequently than females $(3: 1)$ $[3,9]$. Multiple lesions usually in the same limb are found in more than two-thirds of cases [9]. Though the abnormality occurs more frequently on the medial side of the bone, reports involving whole epiphyses have been recorded $[1,5,9$, 11, 12].

There have been many descriptive reports in the literature on the subject, but few on its management. Mouchet and Belot were first to describe it in 1926 and called it "la tarso megalie" [10]. Trevor recognized it as an entity in 1950 and in 1956 Fairbank suggested the name of dysplasia epiphysealis hemimelica as a more suitable terminology $[5,13]$. Most of the reports thereafter present new cases and reviews of the literature. 


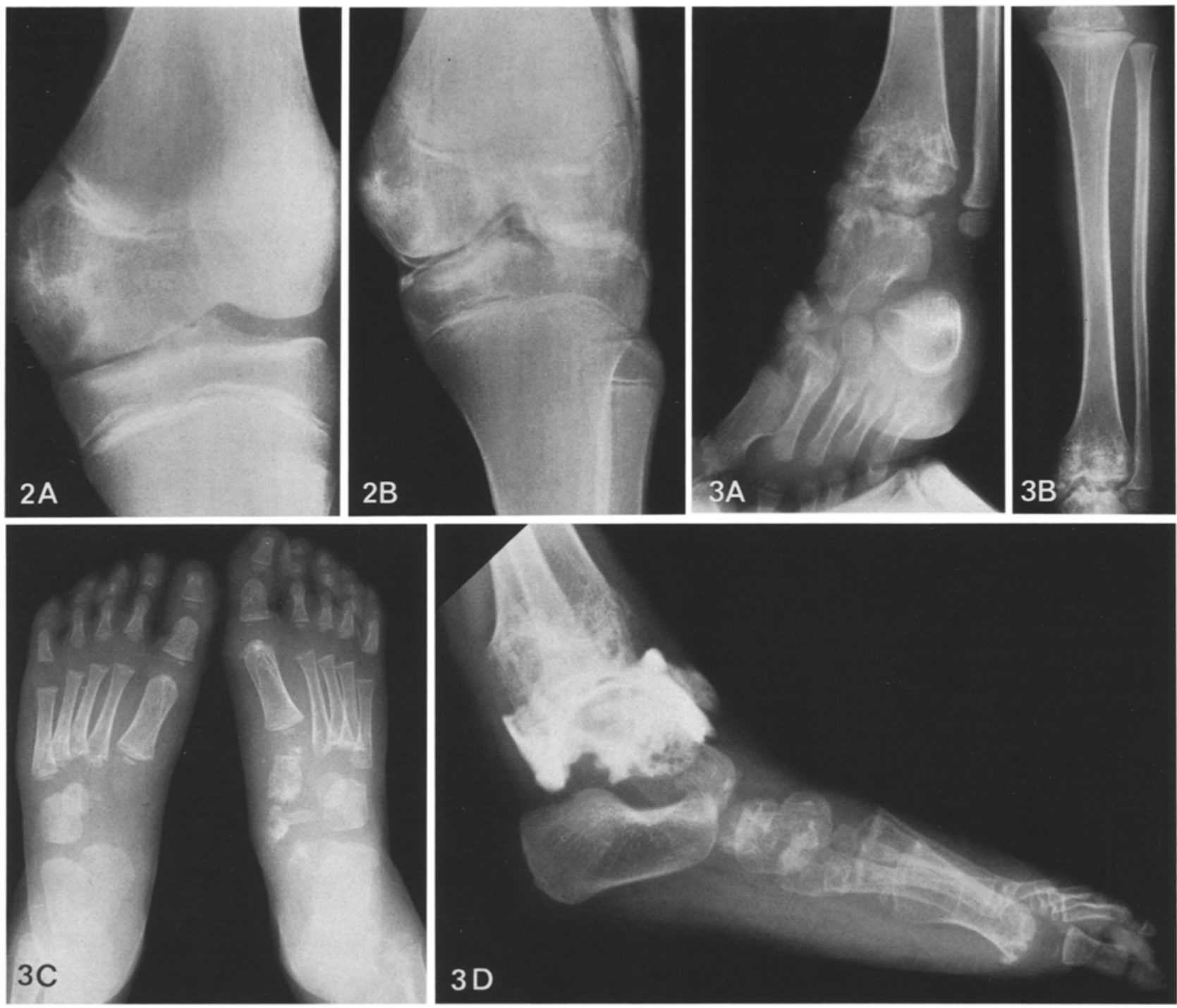

Fig. 2. A An ossified mass is arising from the medial femoral condyle in this 14-year-old boy. B Double contrast arthrogram shows no involvement of the articular surface

Fig. 3. A Three-year-old boy with mass over medial malleolus of left ankle. The distal tibial epiphysis is enlarged with irregular calcification extending into the ankle joint. B Note the linear streaking in the upper tibial metaphysis and the pressure defect at the lower fibula at age four years. $\mathbf{C}$ The left medial cuneiform and navicular were involved. Double ossification centers of middle phalanx of both third toes are also noted. D Lateral view of the double contrast arthrogram demonstrates that both tibial and talar articular surfaces are smooth. The posterior and medial extension are contiguous with the subarticular epiphysis

Discrepancy of limb length has been noted. The affected limb may be longer due to enlargement of several ossification centers and diaphyseal length $[5,9,11]$. It may also be shorter due to early focal closure of the epiphyseal plate $[1,5,9]$. Our first case revealed overgrowth of a limb and the third case revealed limb shortening without closure of the growth plate. The etiology is not readily apparent.

The underlying defect seems to be an abnormality of the regulation of cartilage proliferation in the affected epiphysis, tarsal, or carpal bones resulting in cartilaginous exostoses and cartilage rests in the metaphyses [2]. These patients often come to medical attention because of a localized bump or altered joint mechanics resulting in a valgus; less frequently varus deformity of the lower limb is present.

Radiographically, multiple centers of ossification are seen at the affected site of the epiphysis. Depending on the age at presentation, the ossification center may not have appeared and the carti- 
laginous mass remains unossified. In such cases, particularly those with symptoms such as pain and deformity, information of its extent and involvement of the articular surface is best obtained from a double contrast arthrogram $[4,7,8]$. The procedure is simple to perform, usually requiring only a local anesthetic at the site of injection. The double contrast arthrograms in our cases answered the major clinical questions facing the orthopedic surgeon:

1. Is the articular surface involved?

2. What is the configuration of the articular surface?

3. What is the extent of the cartilaginous mass?

4. What adjacent areas are involved?

5. Is the epiphyseal plate involved?

We were able to demonstrate to the surgeon in each of our cases that the articular surface was smooth and had a normal configuration. The mass mainly involved the subarticular area, with sparing of the epiphyseal plate. This is important as the surgical procedure could then be designed to preserve the articular surface. Preservation of a smooth articular surface is highly desirable as these juxtarticular inequalities can progress to premature osteoarthritis [5]. On fluoroscopy during the arthrogram the joint dynamics are easily demonstrated and recorded on videotape as desired.

\section{References}

1. Azouz EM, Slomic AM, Marton D, Rigault P, Finidori $\mathrm{G}$ (1985) The variable manifestations of dysplasia epiphysealis hemimelica. Pediatr Radiol 15:44

2. Connor JM, Horan FT, Beighton P (1983) Dysplasia epiphysealis hemimelica. J Bone Joint Surg [Br] 65:350

3. D'Angio AJ, Ritvo M, Ulin R (1953) Clinical and roentgen manifestations of tarso-epiphyseal aclasis. AJR 74: 1068

4. Donaldson JS, Sankey HH, Girdany BR, Donaldson WF (1953) Osteochondroma of the distal femoral epiphysis. J Pediatr $43: 212$

5. Fairbank TJ (1956) Dysplasia epiphysealis hemimelica (tarso-epiphysial aclasis). J Bone Joint Surg [Br] 38:237

6. Freiberger RH (1979) The elbow. In: Hudson T (ed) Arthrography. Appleton-Century-Crofts, New York p 261

7. Fisher MR, Hernandez RJ, Poznanski AK, Tachdjian MO (1984) Case report 262. Skeletal Radiol 11:147

8. Goldstein WB (1973) Dysplasia epiphysealis hemimelica with confirmation by knee arthrography. $\mathrm{Br} \mathrm{J}$ Radiol $46: 460$

9. Kettelkamp DB, Campbell CJ, Bonfiglio M (1966) Dysplasia epiphysealis hemimelica. J Bone Joint Surg [Am] 48:746

10. Mouchet A, Belot J (1926) Tarsomegalie. J Radiol Electrol $10: 289$

11. Saxton HM, Wilkinson JA (1964) Hemimelica skeletal dysplasia. J Bone Joint Surg [Br] 46:608

12. Theodoron S, Lanitis A (1968) Dysplasia epiphysealis hemimelica (epiphyseal osteochondromata). Helv Paediatr Acta 2:195

13. Trevor D (1950) Tarso-epiphyseal aclasis. J Bone Joint Surg [Br] 32:204 